\title{
ON ATTAINING THE PRESCRIBED QUALITY OF A CONTROLLED FOURTH ORDER SYSTEM
}

\author{
VLADIMIR KAPUSTYAN *, VYACHESLAV MAKSIMOV**,*** \\ * Department of Mathematical Modeling for Economic Systems \\ National Technical University of Ukraine "Kyiv Polytechnic Institute", 37 Peremohy av., Kyiv 03056, Ukraine \\ e-mail: kapustyanv@ukr.net \\ ${ }^{* *}$ Institute of Mathematics and Mechanics \\ Ural Branch, Russian Academy of Sciences, 16 S. Kovalevskoi str., Ekaterinburg 620990, Russia \\ e-mail: maksimov@imm.uran.ru \\ ${ }^{* * *}$ Chair of Control Systems Modeling \\ Ural Federal University, Ekaterinburg 620002, Russia
}

\begin{abstract}
In this paper, we discuss a method of auxiliary controlled models and its application to solving some robust control problems for a system described by differential equations. As an illustration, a system of nonlinear differential equations of the fourth order is used. A solution algorithm, which is stable with respect to informational noise and computational errors, is presented. The algorithm is based on a combination of online state/input reconstruction and feedback control methods.
\end{abstract}

Keywords: auxiliary models, feedback control, online reconstruction.

\section{Introduction: Problem statement}

A dynamical model connecting the main economic and climatic indices was suggested by Nordhaus (1994). This model consists of various components:

(i) Constant parameters (Nordhaus, 1994, Tables 2.3 and 2.4 , p. 21).

(ii) Functions that are considered (for simplicity of the analysis) exogenous with respect to the model and are given a priori.

(iii) Inner functions that are connected to one another and to exogenous parameters by means of some algebraic and differential equations (Nordhaus, 1994, Tables 2.3 and 2.2).

Consider the following functions: $\mu(t)$, the rate of emission reduction with respect to uncontrollable emissions; $E(t)$, the amount of emissions of Greenhouse Gases (GHGs) $\left(\mathrm{CO}_{2}\right.$ (carbonic acid gas) and chlorine-fluorine carbons only); $M_{1}(t)=(M(t)-590)$, the excess of the mass of GHGs in the atmosphere with respect to the pre-industrial period; $T_{0}(t)$, the average atmospheric temperature (on the Earth's surface); $T_{1}(t)$, the average deep-ocean temperature; $I(t)$, a gross investment; $K(t)$, a capital stock; $F(t)$, an atmospheric radiative forcing from GHGs; $O(t)$, a forcing of exogenous GHGs (i.e., of gases which are considered as uncontrollable; these are all GHGs except $\mathrm{CO}_{2}$ (carbonic acid gas) and chlorine-fluorine carbons); $A(t)$, the level of technology; $\sigma(t)$, the ratio of GHGs emissions to global output; $L(t)$, the population at time $t$, also equal to labor inputs; $Q(t)$, the gross world product.

If we pass from the discrete-time model suggested by various authors to the "continuous" one, then the corresponding equations of the model $\Sigma$ take the form

$$
\begin{aligned}
\dot{T}_{0}(t) & =c_{1} T_{0}(t)+c_{2} T_{1}(t)+c_{3} F(t), \\
\dot{T}_{1}(t) & =c_{4}\left(T_{0}(t)-T_{1}(t)\right), \\
\dot{M}_{1}(t) & =\beta E(t)-\delta_{M} M_{1}(t), \\
\dot{K}(t) & =-\delta_{K} K(t)+I(t), \quad t \in[0, \vartheta],
\end{aligned}
$$

where $t$ is time, $\vartheta$ is the terminal time instant,

$$
F(t)=4.1 \cdot \log _{2}\left(1+\frac{M_{1}(t)}{590}\right)+O(t),
$$




$$
\begin{aligned}
& E(t)=(1-\mu(t)) \sigma(t) Q(t), \\
& Q(t)=\frac{\left(1-b_{1} \mu(t)^{b_{2}}\right)}{\left(1+\theta_{1} T_{0}(t)^{\theta_{2}}\right)} A(t) K(t)^{\gamma} L(t)^{1-\gamma} .
\end{aligned}
$$

Refer to the work of Nordhaus (1994) for more details on model description (Ch. 2) as well as on the physical meaning of coefficients in system (1) (Table 2.3, p. 20).

An initial state of $\Sigma, x(0)=\left\{T_{0}(0), T_{1}(0), M_{1}(0)\right.$, $K(0))\}$, is assumed to be known and given a priori. It is natural to set $T_{0}(0)>0, T_{1}(0)>0$, and $K(0)>0$. Functions $\mu(\cdot)$ and $I(\cdot)$ are considered control inputs determining a strategy of global control of climate and economy. The numerical analysis of the model is performed by Nordhaus (1994). In that work, the direct problem is solved, namely, possible strategies (rules of forming $\mu(\cdot)$ and $I(\cdot))$ are specified, and the system's dynamics are computed. In what follows, functions $\mu$ and $I$, according to Nordhaus (1994), are treated as controls and are denoted by the symbol $u$, i.e., $u=\{\mu, I\}$. We transform the system (1) to the following form (setting small values of $b_{1}=0.0686$ and $\vartheta_{1}=0.00144$ to zero):

$$
\begin{aligned}
\dot{T}_{0}(t)= & c_{1} T_{0}(t)+c_{2} T_{1}(t) \\
& +c_{5} \log _{2}\left(1+\frac{M_{1}(t)}{590}\right)+c_{3} O(t), \\
\dot{T}_{1}(t) & =c_{4}\left(T_{0}(t)-T_{1}(t)\right), \\
\dot{M}_{1}(t) & =E_{1}(t)(1-\mu(t))-\delta_{M} M_{1}(t), \\
\dot{K}(t) & =-\delta_{K} K(t)+I(t), \quad t \in[0, \vartheta],
\end{aligned}
$$

where $c_{5}=4.1 c_{3}$,

$$
E_{1}(t)=E_{1}(t, K)=\beta \sigma(t) A(t) K(t)^{\gamma} L(t)^{1-\gamma} .
$$

In the sequel we consider the system $\Sigma$ of the form (2). The symbol $x(\cdot)=x(\cdot ; x(0), u(\cdot))$ stands for the solution of the system (2) with an initial state $x(0)$ and a control $u(\cdot)=\{\mu(\cdot), I(\cdot)\}$.

Our aim differs from that of Nordhaus (1994). We consider an "inverse" problem consisting in the following. Some system dynamics, i.e., a function $x_{*}(\cdot)=\left\{T_{0 *}(\cdot), T_{1 *}(\cdot), K_{*}(\cdot), M_{1 *}(\cdot)\right\}$ generated by some unknown controls $\mu=\mu_{*}(\cdot)$ and $I=I_{*}(\cdot)$ are given. These controls may be program or feedback controls; the latter is formed, for example, by the rule $\mu_{*}(t)=\mu\left(t, x_{*}(t)\right), I_{*}(t)=I\left(t, x_{*}(t)\right)$. Thus, the functions $x_{*}(\cdot)=\left\{T_{0 *}(\cdot), T_{1 *}(\cdot), K_{*}(\cdot), M_{1 *}(\cdot)\right\}$ satisfy the system of equations

$$
\begin{aligned}
\dot{T}_{0 *}(t)= & c_{1} T_{0 *}(t)+c_{2} T_{1 *}(t) \\
& +c_{5} \log _{2}\left(1+\frac{M_{1 *}(t)}{590}\right)+c_{3} O_{*}(t), \\
\dot{T}_{1 *}(t)= & c_{4}\left(T_{0 *}(t)-T_{1 *}(t)\right), \\
\dot{M}_{1 *}(t)= & E_{1 *}\left(t, K_{*}\right)\left(1-\mu_{*}(t)\right)-\delta_{M} M_{1 *}(t), \\
\dot{K}_{*}(t)= & -\delta_{K} K_{*}(t)+I_{*}(t), \quad t \in[0, \vartheta],
\end{aligned}
$$

where, we emphasize it once again, the functions $\mu_{*}(\cdot)$ and $I_{*}(\cdot)$ are unknown. It is known only that they are subject to restrictions of the form

$$
I_{*}(t) \in\left[I_{-}, I_{+}\right], \quad \mu_{*}(t) \in\left[f_{-}, f_{+}\right] \text {for } t \in[0, \vartheta] .
$$

Here

$$
-\infty<f_{-}<f_{+}<+\infty, \quad 0 \leq I_{-}<I_{+}<+\infty .
$$

The initial state of the system (3), $x_{*}(0)=\left\{T_{0 *}(0)\right.$, $\left.T_{1 *}(0), M_{1 *}(0), K_{*}(0)\right\}$, is assumed to be $x(0)$.

The control problem under discussion may be formulated in the following way. At time instants frequent enough

$\tau_{i} \in \Delta=\left\{\tau_{i}\right\}_{i=0}^{m}, \quad \tau_{i+1}=\tau_{i}+\delta, \quad \tau_{0}=0, \quad \tau_{m}=\vartheta$

values of $T_{0}\left(\tau_{i}\right), T_{1}\left(\tau_{i}\right)$, and $K\left(\tau_{i}\right)$ are inaccurately measured. Results of measurements (vectors $\left\{\xi_{1 i}^{h}, \xi_{2 i}^{h}\right.$, $\left.\xi_{3 i}^{h}\right\} \in \mathbb{R}^{3}$ ) satisfy the inequalities

$$
\begin{aligned}
& \left\{\left(T_{0}\left(\tau_{i}\right)-\xi_{1 i}^{h}\right)^{2}+\left(T_{1}\left(\tau_{i}\right)-\xi_{2 i}^{h}\right)^{2}\right. \\
& \left.+\left(K\left(\tau_{i}\right)-\xi_{3 i}^{h}\right)^{2}\right\} \leq h \\
& +
\end{aligned}
$$

where $h \in(0,1)$ is the level of informational noise. A number $\varepsilon>0$ is given. It is required to construct an algorithm for forming a feedback control,

$$
\begin{gathered}
u=u_{i}^{h}=u\left(\tau_{i} ; x_{*}(\cdot), \xi^{h}(\cdot)\right), \\
t \in\left[\tau_{i}, \tau_{i+1}\right), \quad i \in\{\phi, \ldots, m-1\},
\end{gathered}
$$

that ensures a prescribed quality of the controlled system (2). Namely, the algorithm is to implement the following condition. Whatever unknown possible Lebesgue measurable functions $\mu_{*}(\cdot)$ and $I_{*}(\cdot)$ with the properties (4) may be, the distance between $x^{h}(t)$ and $x_{*}(t)$ at all moments $t \in[0, \vartheta]$ should not exceed the value of $\varepsilon$ provided the values of $h$ and $\delta$ are sufficiently small.

Here $x^{h}(\cdot)=x\left(\cdot ; x(0), u^{h}(\cdot)\right)=\left\{T_{0}^{h}(\cdot), T_{1}^{h}(\cdot)\right.$, $\left.M_{1}^{h}(\cdot), K^{h}(\cdot)\right\}$ is the trajectory of $\Sigma$ generated by the control $u^{h}(\cdot)$ which is formed according to the feedback principle, i.e., $x^{h}(\cdot)$ is the solution of the system (2) with the feedback controls $\mu(\cdot)=\mu^{h}(\cdot)$ and $I(\cdot)=I^{h}(\cdot)$.

This is an informal statement of the problem discussed in the present paper.

In what follows, the symbol $\mathcal{U}$ stands for the set of admissible controls, i.e., the set of Lebesgue measurable functions $u(\cdot)=\{\mu(\cdot), I(\cdot)\}$ such that $\mu(t) \in\left[f_{-}, f_{+}\right]$, $I(t) \in\left[I_{-}, I_{+}\right]$for a.a. $t \in[0, \vartheta]$.

Note that the system (1) is "well-posed". Under this term, we mean the following. First, as is easily seen, in the domain $1+M_{1} / 590>0$, the Carathodory solution of the system (1) is uniquely defined for any $\{\mu(\cdot), I(\cdot)\} \in \mathcal{U}$ and any initial state. Second, the discrete-time model of 
Nordhaus (1994, p. 19) can be obtained from (1) by the discretization of our system via the Euler method with an appropriate step.

One of the approaches to solving the problems of guaranteed control (they are also called positional differential games) for dynamical systems described by ordinary differential equations was developed by Krasovskii and Subbotin (1988). In the work cited above, the cases when the full phase state of a system is inaccurately measured at frequent enough time instants are considered. In the present work, using the approach described by Osipov and Kryazhimskii (1995), the problems of guaranteed control under the measurement of a "part" of a system's phase state (a "part" of coordinates) are investigated (see also Osipov and Kryazhimskii, 1995; Blizorukova and Maksimov, 2006).

To form a control $u$ being a solution to our problem, along with the information on the "part" of coordinates of the solution of the system $\Sigma$ (namely, on the values $\xi_{i}^{h}$ satisfying the inequalities (5)), it is necessary to obtain some additional information on the coordinate $M_{1}$ which is missing. To get such a piece of information during the control process, it is reasonable, following the approach developed by Osipov and Kryazhimskii (1995), Maksimov (2010) as well as Osipov et al. (2011), to introduce an auxiliary controlled system $M$. This system is described by a differential equation (its form is specified below). The equation has an output $w^{h}(t)$ and an input $v^{h}(t)$. The input $v^{h}(\cdot)$ is some new auxiliary control; it should be formed by the feedback principle in such a way that $v^{h}(\cdot)$ "approximates" the unknown coordinate $M_{1}(\cdot)$ in the mean uniform metric. Thus, along with the block of forming the control in the real system (it is called a controller), we need to incorporate into the control contour one more block (it is called an identifier) allowing us to reconstruct the missing coordinate $M_{1}(\cdot)$ in a real time mode. The scheme of algorithms for solving the problem is given in Fig. 1.

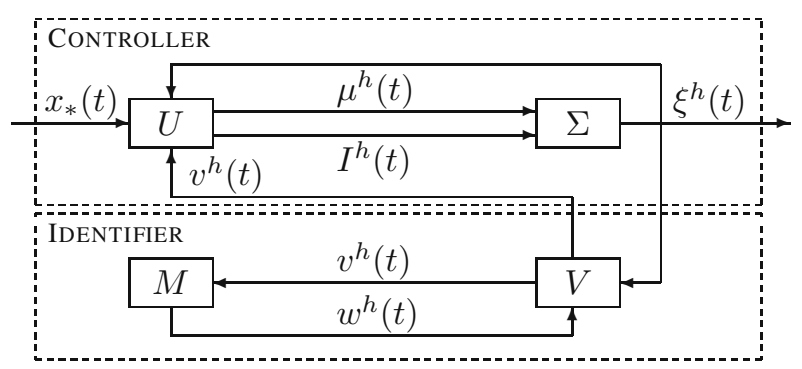

Fig. 1. Scheme of solution algorithms.

To start with an auxiliary dynamical system $M$ (a model) is introduced. This model functioning on the time interval $[0, \vartheta]$ has an input $v^{h}(t)$ and an output $w^{h}(t)$. The model $M$ with its control law $V$ forms the identifier.
Before the algorithm starts, the value $h$ and the partition $\Delta$ with the step $\delta$, as well as the model $M$, are fixed. The process of synchronous feedback control of the systems $\Sigma$ and $M$ is organized on the interval $[0, \vartheta]$. This process is decomposed into $m-1$ identical steps. At the $i$-th step carried out during the time interval $\delta_{i}=\left[\tau_{i}, \tau_{i+1}\right)$, the following actions are fulfilled. First, at the time instant $\tau_{i}$, according to the chosen rules $U$ and $V$, the functions

$$
\begin{array}{r}
v^{h}(t)=v_{i}^{h} \in V\left(\tau_{i}, \xi_{i}^{h}, w^{h}\left(\tau_{i}\right)\right), \quad t \in \delta_{i}, \\
u^{h}(t)=u_{i}^{h}=\left\{\mu_{i}^{h}, I_{i}^{h}\right\} \\
\in U\left(\tau_{i}, v_{i}^{h}, \xi_{i}^{h}, x_{*}\left(\tau_{i}\right)\right) \\
\subset\left[I_{-}, I_{+}\right] \times\left[f_{-}, f_{+}\right],
\end{array}
$$

are calculated by measurements $\xi_{i}^{h}$ and $w^{h}\left(\tau_{i}\right)$. Then (till the moment $\tau_{i+1}$ ) the control $u=u^{h}(t), \tau_{i} \leq t<\tau_{i+1}$, is fed onto the input of the system $\Sigma$ and the control $v=$ $v^{h}(t), \tau_{i} \leq t<\tau_{i+1}$, onto the input of the model $M$. The values $\xi_{i+1}^{h}$ and $w^{h}\left(\tau_{i+1}\right)$ are the results of the work of the algorithm at the $i$-th step. The procedure stops at the moment $\vartheta$.

Thus, the complexity of solving these problems is reduced to an appropriate choice of a model $M$ as well as functions $U$ and $V$. Consequently, a mathematical statement of the problem may be formulated as follows. In the sequel, a family of partitions

$$
\begin{gathered}
\Delta_{h}=\left\{\tau_{i, h}\right\}_{h=0}^{m_{h}}, \quad \tau_{i+1, h}=\tau_{i, h}+\delta(h), \\
\tau_{0, h}=0, \quad \tau_{m_{h}, h}=\vartheta,
\end{gathered}
$$

of the interval $[0, \vartheta]$ is assumed to be fixed.

Problem of robust control. It is required to specify differential equations of the model $M$ in the form

$$
\begin{gathered}
\dot{w}^{h}(t)=f_{1}\left(\xi_{i}^{h}, w^{h}\left(\tau_{i}\right), v^{h}(t)\right), \\
t \in \delta_{h, i}=\left[\tau_{i}, \tau_{i+1}\right), \quad \tau_{i}=\tau_{i, h}, \\
w^{h}(0)=w_{0}^{h}, \quad w^{h}(t) \in \mathbb{R},
\end{gathered}
$$

and the rule of choosing controls $v_{i}^{h}(t)$ in the model 8 and $u_{i}^{h}(t)$ in the system (2) at the moments $\tau_{i}$ being a mapping of the form (6), (7) such that the inequality

$$
\max _{t \in[0, \vartheta]}\left\|x^{h}(t)-x_{*}(t)\right\| \leq \varepsilon
$$

holds for $h \in\left(0, h_{*}(\varepsilon)\right)$ and $\delta=\delta(h) \in\left(0, \delta\left(h_{*}(\varepsilon)\right)\right.$.

Here, the symbol $\|\cdot\|$ denotes the Euclidean norm of a vector, $x^{h}(\cdot)=x\left(\cdot ; x(0), u^{h}(\cdot)\right)$ is the trajectory of $\Sigma$ generated by the control $u^{h}(\cdot)$ that is formed according to the feedback principle (6), (7).

After writing down the model equations (8) and the rules for forming controls (6) and (7), the sequence of actions which should be performed to provide "closeness" of a trajectory of system (2) and a given trajectory 
$x_{*}(\cdot)$ (i.e., the inequality (9), is similar to the sequence described above (see also the end of Section 3, where the corresponding algorithm is presented).

From the above scheme, it follows that one of the important steps of the algorithm is the reconstruction of the unmeasured coordinate $M_{1}$ in "real-time" mode. It should be noted that the problems of online reconstruction of states/inputs were considered from the position of the approach above by Keesman and Maksimov (2008), Blizorukova and Maksimov (2010) as well as Osipov et al. (2011) for some classes of systems described by ordinary differential equations of the second and third orders. The algorithms designed in these works allowed reconstructing the corresponding characteristics in the $L_{2}$-metric. The algorithm for reconstructing the coordinate $M_{1}$ described in Section 2 allows us to get an approximation of $M_{1}$ in the uniform metric (the metric of space $C$ of continuous functions).

Let the symbol $X(\cdot)$ denote the set of solutions of the system (2), i.e., $X(\cdot)=\{x(\cdot): x(\cdot)=x(\cdot ; x(0), u(\cdot))=$ $\left.\left\{T_{0}(\cdot), T_{1}(\cdot), M_{1}(\cdot), K(\cdot)\right\}, u(\cdot) \in \mathcal{U}\right\}$. We assume that the following condition is fulfilled.

\section{Condition 1.}

$$
\begin{aligned}
d_{*} & =\inf \left\{\min _{t \in[0, \vartheta]}\left(1+\frac{M_{1}(t)}{590}\right):\right. \\
x(\cdot) & \left.=\left\{T_{0}(\cdot), T_{1}(\cdot), M_{1}(\cdot), K(\cdot)\right\} \in X(\cdot)\right\}>1 .
\end{aligned}
$$

In addition, the functions $\sigma(t), A(t), L(t)$, and $O(t)$ are considered to be known and continuous.

\section{Algorithm for reconstructing $M_{1}(\cdot)$}

First, we specify the algorithm for reconstructing $M_{1}(\cdot)$, which will be applied for solving the problem in question. Namely, we describe the identifier (see Fig. 1), i.e., specify the equation of the model $M$ and its control law $V$. To substantiate this algorithm, we use ideas of Osipov and Kryazhimskii (1995), Osipov et al. (2011), Blizorukova and Maksimov (2006) as well as Maksimov (2013).

Introduce the notation

$$
\begin{gathered}
T(t)=\left\{T_{0}(t), T_{1}(t)\right\}, \\
f(t, T(t))=c_{1} T_{0}(t)+c_{2} T_{1}(t)+c_{3} O(t), \\
\tilde{u}(t)=\log _{2}\left(1+\frac{M_{1}(t)}{590}\right) .
\end{gathered}
$$

Here $x(\cdot)=\left\{T_{0}(\cdot), T_{1}(\cdot), M_{1}(\cdot), K(\cdot)\right\}$ is an arbitrary element of the set $X(\cdot)$. In this case, the first equation of the system (2) is rewritten in the form

$$
\dot{T}_{0}(t)=f(t, T(t))+c_{5} \tilde{u}(t) .
$$

Note that one can specify a number $M_{*}>0$ such that the following inequalities are valid:

$$
\begin{gathered}
\|\dot{T}(t)\| \leq M_{*} \quad \text { for a.a. } t \in[0, \vartheta], \\
\left|f(t, T(t))-f\left(\tau_{i}, \xi_{i}^{h}\right)\right| \leq M_{*}(\delta+h+\omega(\delta))
\end{gathered}
$$

for $t \in \delta_{i}=\left[\tau_{i}, \tau_{i+1}\right)$. Here the symbol $|\cdot|$ stands for the absolute value of a number, $\tau_{i}=\tau_{i, h}, \omega(\delta)$ is the continuity modulus of the function $t \rightarrow O(t), t \in[0, \vartheta]$, i.e.,

$$
\omega(\delta)=\sup \{|O(t)-O(t-\delta)|: t \in[\delta, \vartheta]\} .
$$

The inequality (11) is a consequence of (5) and (10).

We fix a family $\Delta_{h}$ of partitions of the interval $[0, \vartheta]$ and some auxiliary function $\alpha(h):(0,1) \rightarrow(0,1)$. As the model $M$, we take a linear system described by a scalar differential equation of the form

$$
\dot{w}^{h}(t)=f\left(\tau_{i}, \xi_{i}^{h}\right)+c_{5} v^{h}(t)
$$

for a.a. $t \in \delta_{i}=\left[\tau_{i}, \tau_{i+1}\right), i \in\{0, \ldots, m-1\}, \tau_{i}=\tau_{i, h}$, $m=m_{h}$, with the initial condition

$$
w^{h}(0)=T_{0}(0) .
$$

Let

$$
\begin{aligned}
v^{h}(t) & =v_{i}^{h} \\
& \in V\left(\tau_{i}, \xi_{i}^{h}, w^{h}\left(\tau_{i}\right)\right) \\
& =-\frac{1}{\alpha} c_{5}\left[w^{h}\left(\tau_{i}\right)-\xi_{1 i}^{h}\right]
\end{aligned}
$$

for a. a. $t \in \delta_{i}$. The control $v^{h}(t)$ in Eqn. (12) is found from (13). Thus, the model control is specified by the feedback principle (see (6)). Consequently, Eqn. (12) takes the form

$$
\dot{w}^{h}(t)=f\left(\tau_{i}, \xi_{i}^{h}\right)-\frac{1}{\alpha} c_{5}^{2}\left[w^{h}\left(\tau_{i}\right)-\xi_{1 i}^{h}\right]
$$

for a.a. $t \in \delta_{i}$.

Let us describe the algorithm for reconstructing the unmeasured coordinate $M_{1}(\cdot)$ in real time mode. Before the algorithm starts, we fix a value $h \in(0,1)$ and a partition $\Delta_{h}$. The work of the algorithm is decomposed into $m-1$ identical steps. At the $i$-th step carried out during the time interval $\delta_{i}=\left[\tau_{i}, \tau_{i+1}\right), \tau_{i}=\tau_{i, h}$, the following actions are made. First, at the moment $\tau_{i}$, the control $v^{h}(t)$ is calculated by (13). This control is fed to the input of the model (12) on the interval $\left[\tau_{i}, \tau_{i+1}\right)$. Under the action of this control, the model passes from the state $w^{h}\left(\tau_{i}\right)$ to the state $w^{h}\left(\tau_{i+1}\right)=$ $w^{h}\left(\tau_{i+1} ; \tau_{i}, w^{h}\left(\tau_{i}\right), v_{i}^{h}\right)$. The work of the algorithm stops at the moment $\vartheta$.

Denote by $\Xi(x(\cdot), h)$ the set of admissible measurements, i.e., the set of all piece wise constant functions $\xi^{h}(\cdot) \rightarrow \mathbb{R}^{3}, \xi^{h}(t)=\xi_{i}^{h}$ for $t \in\left[\tau_{i}, \tau_{i+1}\right)$, $\tau_{i}=\tau_{i, h}$, satisfying the inequalities (5). Here $\xi_{i}^{h}=\left\{\xi_{1 i}^{h}, \xi_{2 i}^{h}, \xi_{3 i}^{h}\right\}$. 
Lemma 1. Let the conditions

$$
\begin{aligned}
\alpha(h) & \rightarrow 0, \quad \delta(h) \rightarrow 0, \\
\delta(h) \alpha^{-1}(h) \rightarrow 0, \quad h \alpha^{-1}(h) & \rightarrow 0 \text { as } h \rightarrow 0
\end{aligned}
$$

be fulfilled. Then, uniformly in all $x(\cdot) \in X(\cdot), h \in(0,1)$, $\xi^{h}(\cdot) \in \Xi(x(\cdot), h), i \in\left\{0, \ldots, m_{h}-1\right\}$, the inequalities

$$
\int_{\tau_{i}}^{\tau_{i+1}}\left|\dot{w}^{h}(s)\right| \mathrm{d} s \leq C \delta
$$

are valid. Here $C=$ const $>0, \delta=\delta(h)$, and $\tau_{i}=\tau_{i, h}$.

Proof. Taking into account (14), we deduce

$$
\begin{aligned}
\frac{\mathrm{d}}{\mathrm{d} t} & {\left[w^{h}(t)-T_{0}(t)\right] } \\
= & f\left(\tau_{i}, \xi_{i}^{h}\right)-\frac{1}{\alpha} c_{5}^{2}\left[w^{h}\left(\tau_{i}\right)-\xi_{1 i}^{h}\right] \\
& -f(t, T(t))-c_{5} \tilde{u}(t) \\
= & -\frac{1}{\alpha} c_{5}^{2}\left[w^{h}(t)-T_{0}(t)\right]+\Psi_{h}^{(1)}(t)
\end{aligned}
$$

for a. a. $t \in \delta_{i}$, and

$$
w^{h}(0)=T_{0}(0)
$$

where

$$
\begin{aligned}
\Psi_{h}^{(1)}(s)= & \Psi_{h}(s)+\frac{1}{\alpha} c_{5}^{2}\left[w^{h}(s)-w^{h}\left(\tau_{i}\right)\right] \\
\Psi_{h}(s)= & -\frac{1}{\alpha} c_{5}^{2}\left[T_{0}(s)-\xi_{1 i}^{h}\right]+\left[f\left(\tau_{i}, \xi_{i}^{h}\right)\right. \\
& -f(s, T(s))]-c_{5} \tilde{u}(s) \text { for a.a. } s \in \delta_{i} .
\end{aligned}
$$

Here, by virtue of (5), (10), (11) and (15), the family of functions $\Psi_{h}(\cdot)$ is bounded,

$$
\left|\Psi_{h}(s)\right| \leq M^{(1)} \quad \text { for a.a. } t \in[0, \vartheta],
$$

uniformly with respect to all $h \in(0,1)$. Further, we have

$$
\begin{aligned}
w^{h}(t) & -T_{0}(t) \\
& =\int_{0}^{t} e^{-\frac{1}{\alpha} c_{5}{ }^{2}(t-s)} \Psi_{h}^{(1)}(s) \mathrm{d} s, \quad t \in[0, \vartheta] .
\end{aligned}
$$

Let

$$
\begin{array}{r}
\mu(t)=\max _{0 \leq \tau \leq t}\left|w^{h}(\tau)-T_{0}(\tau)\right|, \\
f_{h}(t)=f\left(\tau_{i}, \xi_{i}^{h}\right) \quad \text { for } t \in \delta_{i} .
\end{array}
$$

Then the following estimations are true:

$$
\begin{aligned}
& \frac{1}{\alpha} c_{5}{ }^{2} \int_{\tau_{i}}^{\tau_{i+1}}\left|\dot{w}^{h}(s)\right| \mathrm{d} s \\
& \leq \frac{K_{0}}{\alpha} \int_{\tau_{i}}^{\tau_{i+1}}\left|f_{h}(s)-\frac{1}{\alpha} c_{5}{ }^{2}\left[w^{h}\left(\tau_{i}\right)-\xi_{1 i}^{h}\right]\right| \mathrm{d} s \\
& \leq K_{1} \frac{\delta}{\alpha}+K_{2} \frac{\delta}{\alpha^{2}}\left(\mu\left(\tau_{i}\right)+h\right), \\
& \mu\left(\tau_{i}\right) \leq \mu\left(\tau_{i+1}\right) .
\end{aligned}
$$

Note that

$$
\begin{aligned}
& \left|\Psi_{h}^{(1)}(t)\right| \\
& \leq\left|\Psi_{h}(t)\right|+\frac{1}{\alpha} c_{5}{ }^{2} \int_{\tau_{i}}^{\tau_{i+1}}\left|\dot{w}^{h}(s)\right| \mathrm{d} s \quad \text { for } t \in \delta_{i} .
\end{aligned}
$$

Thus, taking into account (18)-20), we obtain

$$
\begin{aligned}
\mu(t) \leq & K_{3}\left(\frac{\delta}{\alpha}+\frac{\delta}{\alpha^{2}} \mu\left(\tau_{i}\right)+\frac{\delta h}{\alpha^{2}}\right) \int_{0}^{t} e^{-\frac{1}{\alpha} c_{5}^{2}(t-s)} \mathrm{d} s \\
& +\int_{0}^{t} e^{-\frac{1}{\alpha} c_{5}^{2}(t-s)}\left|\Psi_{h}(s)\right| \mathrm{d} s, \quad t \in \delta_{i} .
\end{aligned}
$$

Using (17), we derive

$$
\int_{0}^{t} e^{-\frac{1}{\alpha} c_{5}^{2}(t-s)}\left|\Psi_{h}(s)\right| \mathrm{d} s \leq K_{4} \int_{0}^{t} e^{-\frac{1}{\alpha} c_{5}^{2}(t-s)} \mathrm{d} s .
$$

It is easily seen that

$$
\begin{aligned}
\int_{0}^{t} e^{-\frac{1}{\alpha} c_{5}^{2}(t-s)} \mathrm{d} s & \\
= & \left.\frac{\alpha}{c_{5}^{2}} e^{-\frac{c_{5}^{2}}{\alpha}(t-s)}\right|_{0} ^{t} \\
& =\frac{\alpha}{c_{5}^{2}}\left(1-e^{-\frac{c_{5}^{2}}{\alpha} t}\right) \leq K_{5} \alpha .
\end{aligned}
$$

Using (22) and (23), we obtain

$$
\int_{0}^{t} e^{-\frac{1}{\alpha} c_{5}^{2}(t-s)}\left|\Psi_{h}(s)\right| \mathrm{d} s \leq K_{6} \alpha .
$$

Taking into account (21), (17), (24) and assuming $t=\tau_{i}$, we have

$$
\left(1-\frac{K_{3} K_{5} \delta}{\alpha}\right) \mu\left(\tau_{i}\right) \leq K_{7}\left(\alpha+\delta+\frac{\delta h}{\alpha}\right) .
$$


Therefore, for sufficiently small $h$ (for example, such that $1-K_{3} K_{5} \delta / \alpha \geq 1 / 2$ ), we obtain

$$
\mu\left(\tau_{i}\right) \leq K_{8}\left(\alpha+\delta+\frac{\delta h}{\alpha}\right) \leq K_{9}(\alpha+\delta)
$$

(see (15)). From (19) it follows that

$$
\int_{\tau_{i}}^{\tau_{i+1}}\left|\dot{w}^{h}(s)\right| \mathrm{d} s \leq K_{10}\left\{\delta+\frac{\delta}{\alpha}\left(\mu\left(\tau_{i}\right)+h\right)\right\} .
$$

In addition, by virtue of (25), we derive

$$
\begin{aligned}
\delta+\frac{\delta}{\alpha}\left(\mu\left(\tau_{i}\right)+h\right) & \leq \delta+K_{8} \frac{\delta}{\alpha}\left(\alpha+h+\delta+\frac{\delta h}{\alpha}\right) \\
& \leq K_{11} \delta
\end{aligned}
$$

Therefore,

$$
\int_{\tau_{i}}^{\tau_{i+1}}\left|\dot{w}^{h}(s)\right| \mathrm{d} s \leq K_{12} \delta .
$$

The inequality (16) is established and the lemma is proved.

Lemma 2. Let the conditions (15) be fulfilled. Let $\delta^{\gamma}(h) \alpha^{-1}(h) \rightarrow+\infty($ for some $\gamma \in(0,1))$ as $h \rightarrow 0$ and

$$
u_{e}^{h}(t)= \begin{cases}\tilde{u}(0), & t \in\left[0, \delta^{\gamma}\right) \\ v^{h}(t), & t \in\left[\delta^{\gamma}, \vartheta\right]\end{cases}
$$

Then the inequality

$$
\begin{aligned}
& \sup _{t \in[0, \vartheta]}\left|u_{e}^{h}(t)-\tilde{u}(t)\right| \\
& \quad \leq d_{1}^{0} \alpha(h)+d_{2}^{0}(h+\delta(h)) \alpha^{-1}(h)+d_{3}^{0} \omega(\delta(h)) \\
& \quad+d_{4}^{0} \alpha(h) \delta^{-\gamma}(h)+d_{5}^{0} \delta^{\gamma}(h)
\end{aligned}
$$

is valid. Here the constants $d_{j}^{0}, j \in 1, \ldots, 5$, do not depend on $h \in(0,1)$.

Proof. It is easily seen that the equality

$$
\begin{aligned}
& \frac{1}{\alpha} c_{5}^{2}\left[w^{h}(t)-T_{0}(t)\right] \\
& =\int_{0}^{t}\left(\frac{d}{\mathrm{~d} s} e^{-\frac{1}{\alpha} c_{5}^{2}(t-s)}\right) \Psi_{h}^{(1)}(s) \mathrm{d} s \\
& =-\int_{0}^{t}\left(\frac{\mathrm{d}}{\mathrm{d} s} e^{-\frac{1}{\alpha} c_{5}^{2}(t-s)}\right) c_{5} \tilde{u}(s) \mathrm{d} s \\
& \quad+\sum_{j=1}^{3} \int_{0}^{t}\left(\frac{\mathrm{d}}{\mathrm{d} s} e^{-\frac{1}{\alpha} c_{5}^{2}(t-s)}\right) \gamma_{\delta}^{(j)}(s) \mathrm{d} s
\end{aligned}
$$

is true. Here

$$
\begin{aligned}
& \gamma_{\delta}^{(1)}(s)=\frac{1}{\alpha} c_{5}^{2}\left[w^{h}(s)-w^{h}\left(\tau_{i}\right)\right], \\
& \gamma_{\delta}^{(2)}(s)=-\frac{1}{\alpha} c_{5}^{2}\left[T_{0}(s)-\xi_{1 i}^{h}\right], \\
& \gamma_{\delta}^{(3)}(s)=f\left(\tau_{i}, \xi_{i}^{h}\right)-f(s, T(s))
\end{aligned}
$$

for a.a. $s \in \delta_{i}$. Using (16) we conclude that

$$
\left|\gamma_{\delta}^{(1)}(s)\right| \leq C_{1} \frac{\delta}{\alpha}, \quad s \in[0, \vartheta]
$$

From (5) and (10) it follows that

$$
\left|\gamma_{\delta}^{(2)}(s)\right| \leq C_{2} \frac{\delta+h}{\alpha}, \quad s \in[0, \vartheta] .
$$

Using 111 we have

$$
\left|\gamma_{\delta}^{(3)}(s)\right| \leq M_{*}(\delta+h+\omega(\delta)), \quad s \in[0, \vartheta] .
$$

In this case, from (27)-29), 23), 24) we deduce that

$$
\begin{aligned}
& \left|\sum_{j=1}^{3} \int_{0}^{t}\left(\frac{\mathrm{d}}{\mathrm{d} s} e^{-\frac{1}{\alpha} c_{5}{ }^{2}(t-s)}\right) \gamma_{\delta}^{(j)}(s) \mathrm{d} s\right| \\
& \quad \leq \varrho(h, \alpha, \delta)=C_{3}\left(\delta+h+\omega(\delta)+\frac{\delta+h}{\alpha}\right) .
\end{aligned}
$$

Integrating by parts the first term on the right-hand side of (26), we obtain

$$
\begin{aligned}
-\int_{0}^{t}\left(\frac{\mathrm{d}}{\mathrm{d} s} e^{-\frac{1}{\alpha} c_{5}{ }^{2}(t-s)}\right) c_{5} \tilde{u}(s) \mathrm{d} s & \\
= & e^{-\frac{1}{\alpha} c_{5}^{2} t} c_{5} \tilde{u}(0)-c_{5} \tilde{u}(t) \\
& +\int_{0}^{t} e^{-\frac{1}{\alpha} c_{5}^{2}(t-s)} c_{5} \dot{\tilde{u}}(s) \mathrm{d} s .
\end{aligned}
$$

From (5), 10), 16) (see Lemma 1), we have that for a.a. $t \in \delta_{i}$

$$
\begin{aligned}
& \left|\frac{1}{\alpha} c_{5}^{2}\left\{\left[w^{h}(t)-T_{0}(t)\right]-\left[w^{h}\left(\tau_{i}\right)-\xi_{1 i}^{h}\right]\right\}\right| \\
& \leq \frac{C_{4}}{\alpha}\left\{\int_{\tau_{i}}^{\tau_{i+1}}\left|\dot{w}^{h}(s)\right| \mathrm{d} s+h+\delta\right\} \leq C_{5} \frac{h+\delta}{\alpha} .
\end{aligned}
$$

By virtue of the boundedness of $\dot{\tilde{u}}(\cdot)(\dot{\tilde{u}}(\cdot) \in$ $L_{\infty}([0, \vartheta] ; \mathbb{R})$ (see Condition 1), using (24), we derive the inequality

$$
\left|\int_{0}^{t} e^{-\frac{1}{\alpha} c_{5}^{2}(t-s)} c_{5} \dot{\tilde{u}}(s) \mathrm{d} s\right| \leq C_{6} \alpha .
$$


Therefore, using this inequality as well as 26, 30 - 32 and (15), we obtain for $t \in \delta_{i}$

$$
\begin{aligned}
\left|\frac{1}{\alpha} c_{5}{ }^{2}\left[w^{h}\left(\tau_{i}\right)-T_{0}\left(\tau_{i}\right)\right]-c_{5} \tilde{u}(t)\right| \\
\leq \varrho(h, \delta, \alpha)+C_{5} \frac{h+\delta}{\alpha} \\
+C_{6} \alpha+\left|e^{-\frac{1}{\alpha} c_{5}^{2} t} c_{5} \tilde{u}(0)\right| .
\end{aligned}
$$

Note that

$$
e^{-c_{*} \frac{\delta \gamma}{\alpha}} \leq \frac{\alpha}{c_{*} \delta \gamma} \quad \text { for } c_{*}>0
$$

The statement of the lemma follows from (33), since, by virtue of the boundedness of $M_{1}(\cdot)$, the inequalities

$$
\begin{aligned}
\left|\tilde{u}(t)-u_{e}^{h}(t)\right| & \leq c \delta^{\gamma} \quad \text { for a.a. } t \in\left[0, \delta^{\gamma}\right), \\
\left|\tilde{u}(t)-u_{e}^{h}(t)\right| & \leq d_{1}^{0} \alpha+d_{2}^{0} \frac{h+\delta}{\alpha}+d_{3}^{0} \omega(\delta) \\
& +d_{4}^{0} \frac{\alpha}{\delta^{\gamma}} \quad \text { for a.a. } t \in\left[\delta^{\gamma}, \vartheta\right]
\end{aligned}
$$

are valid. The lemma is proved.

Introduce the notation

$$
\tilde{u}_{*}^{h}(t)=590\left(2^{u_{e}^{h}(t)}-1\right) .
$$

The following theorem is true.

Theorem 1. Under the conditions of Lemma 2, the inequality

$$
\begin{aligned}
& \sup _{t \in[0, \vartheta]}\left|\tilde{u}_{*}^{h}(t)-M_{1}(t)\right| \leq \nu(h, \delta(h), \alpha(h)) \\
& =d_{1} \alpha(h)+d_{2}(h+\delta(h)) \alpha^{-1}(h)+d_{3} \omega(\delta(h)) \\
& \quad+d_{4} \alpha(h) \delta^{-\gamma}(h)+d_{5} \delta^{\gamma}(h)
\end{aligned}
$$

holds. Here the constants $d_{j}, j \in\{1, \ldots, 5\}$, do not depend on $h \in(0,1)$.

The theorem follows from Lemma 2 and the inequality

$$
\left|\tilde{u}_{*}^{h}(t)-M_{1}(t)\right| \leq 590\left|2^{u_{e}^{h}(t)}-2^{\tilde{u}(t)}\right| .
$$

\section{Algorithm for the control problem}

Let us turn to the description of the algorithm for solving the control problem in question. From the above, it is necessary to specify the model (8) and control laws $U$ (7) and $V$ (6), providing the inequality (9).

We fix a family $\Delta_{h}$ of partitions of the interval $[0, \vartheta]$ and some function $\alpha(h):(0,1) \rightarrow(0,1)$. Let the family $\Delta_{h}$ and the function $\alpha(h)$ be such that the following condition holds
Condition 2. We have

$$
\begin{gathered}
\alpha(h) \rightarrow 0, \quad \delta(h) \rightarrow 0, \quad \delta(h) \alpha^{-1}(h) \rightarrow 0, \\
h \alpha^{-1}(h) \rightarrow 0, \quad \alpha^{-1}(h) \delta^{\gamma}(h) \rightarrow+\infty \quad \text { as } h \rightarrow 0
\end{gathered}
$$

for some $\gamma \in(0,1)$.

Let the model (8) be of form (12), i.e.,

$$
\dot{w}^{h}(t)=f\left(\tau_{i}, \xi_{i}^{h}\right)+c_{5} v^{h}(t)
$$

for a.a. $t \in \delta_{i}=\left[\tau_{i}, \tau_{i+1}\right), i \in\{0, \ldots, m-1\}, \quad \tau_{i}=$ $\tau_{i, h}, \quad m=m_{h}$, with the initial condition

$$
w^{h}(0)=T_{0}(0)
$$

Let rules $U$ (7) and $V$ (6) for forming the controls $u_{i}^{h}$ and $v_{i}^{h}$ be as follows:

$$
\begin{array}{r}
v_{i}^{h}=V\left(\tau_{i}, \xi_{i}^{h}, w^{h}\left(\tau_{i}\right)\right)=-\frac{1}{\alpha} c_{5}\left[w^{h}\left(\tau_{i}\right)-\xi_{1 i}^{h}\right], \\
u_{i}^{h}=\left\{\mu^{h}\left(\tau_{i}\right), I^{h}\left(\tau_{i}\right)\right\}=U\left(\tau_{i}, v_{i}^{h}, \xi_{i}^{h}, x_{*}\left(\tau_{i}\right)\right)
\end{array}
$$

for $t \in \delta_{i}$. Here

$$
\begin{aligned}
& I^{h}\left(\tau_{i}\right)= \arg \min \left\{\left(\xi_{3 i}^{h}-K_{*}\left(\tau_{i}\right)\right) I: I \in\left[I_{-}, I_{+}\right]\right\}, \\
& \mu^{h}\left(\tau_{i}\right)= \arg \min \left\{E_{1}\left(\tau_{i}, K_{*}\right)\right. \\
&\left.\times\left(M_{1 *}\left(\tau_{i}\right)-\tilde{u}_{*}^{h}\left(\tau_{i}\right)\right) \mu: \mu \in\left[f_{-}, f_{+}\right]\right\}, \\
& \tilde{u}_{*}^{h}\left(\tau_{i}\right)=590\left(2^{u_{\varepsilon}^{h}\left(\tau_{i}\right)}-1\right), \\
& u_{\varepsilon}^{h}\left(\tau_{i}\right)= \begin{cases}\log _{2}\left(1+\frac{M_{1}(0)}{590}\right) & \text { if } \tau_{i} \leq \delta^{\gamma}(h), \\
v_{i}^{h} & \text { otherwise. }\end{cases}
\end{aligned}
$$

In what follows, we need the lemma given below.

Lemma 3. (Maksimov, 2011) Let the function $\varepsilon(t)$ be nonpositive for $t \in T$ and, for all $i \in\{0, \ldots, m-1\}$, satisfy the inequalities

$$
\varepsilon\left(\tau_{i+1}\right) \leq \varepsilon\left(\tau_{i}\right)(1+\beta \delta)+\int_{\tau_{i}}^{\tau_{i+1}}|\varphi(t)| \mathrm{d} t,
$$

where $\tau_{i} \in \Delta, \beta=$ const $>0$, and $\varphi(\cdot) \in L_{1}(T ; \mathbb{R})$. Then

$$
\varepsilon\left(\tau_{i}\right) \leq\left(\varepsilon\left(t_{0}\right)+\int_{t_{0}}^{\tau_{i}}|\varphi(t)| \mathrm{d} t\right) \exp \left(\beta\left(\tau_{i}-t_{0}\right)\right) .
$$

Introduce the following condition.

\section{Condition 3. The inequalities}

$$
0<C^{(1)}<K_{*}(t)<C^{(2)}<+\infty \quad \text { for } t \in[0, \vartheta]
$$

are valid. 
Theorem 2. For any $\varepsilon>0$, one can specify $h_{*}(\varepsilon) \in(0,1)$ such that, for all $h \in\left(0, h_{*}(\varepsilon)\right)$ and $\delta(h) \in\left(0, \delta\left(h_{*}(\varepsilon)\right)\right)$, the inequality (9) holds, if the model $M$ is given by Eqn. (34), the strategies $V$ and $U$ are taken in the form (6), (7), (35)-(37).

Proof. First, estimate the variation in the value

$$
\varepsilon_{1}(t)=\left|K_{*}(t)-K^{h}(t)\right|^{2}, \quad t \in[0, \vartheta] .
$$

It is easily seen that for $t \in \delta_{i}=\left[\tau_{i}, \tau_{i+1}\right)$ the following inequality is true:

$$
\begin{aligned}
\varepsilon_{1}(t) \leq & \varepsilon_{1}\left(\tau_{i}\right)+\delta(h) \int_{\tau_{i}}^{t}\left|\dot{K}_{*}(\tau)-\dot{K}^{h}(\tau)\right|^{2} \mathrm{~d} \tau \\
& +\int_{\tau_{i}}^{t} \nu_{i}(\tau) \mathrm{d} \tau .
\end{aligned}
$$

Here

$\nu_{i}(\tau)=2\left(K_{*}\left(\tau_{i}\right)-K^{h}\left(\tau_{i}\right)\right)\left(\dot{K}_{*}(\tau)-\dot{K}^{h}(\tau)\right), \quad \tau \in \delta_{i}$.

Consider the value $\nu_{i}(t)$. We have for $t \in \delta_{i}$

$$
\nu_{i}(t)=\sum_{j=1}^{4} \nu_{j i}(t), \quad t \in \delta_{i},
$$

where

$$
\begin{aligned}
& \nu_{1 i}(t)=2 \beta_{i}^{(1)} \delta_{K}\left(K_{*}\left(\tau_{i}\right)-K_{*}(t)\right), \\
& \nu_{2 i}(t)=2 \beta_{i}^{(1)} \delta_{K}\left(K^{h}(t)-K^{h}\left(\tau_{i}\right)\right), \\
& \nu_{3 i}(t)=2 \delta_{K} \varepsilon_{1}\left(\tau_{i}\right) \\
& \nu_{4 i}(t)=2 \beta_{i}^{(1)} \delta_{K}\left(I_{*}(t)-I^{h}\left(\tau_{i}\right)\right), \\
& \beta_{i}^{(1)}=K_{*}\left(\tau_{i}\right)-K^{h}\left(\tau_{i}\right) .
\end{aligned}
$$

Estimate each term on the right-hand side of the equality (39). From (2) and (4) it follows that

$$
\nu_{1 i}(t) \leq C_{1} \delta, \quad \nu_{2 i}(t) \leq C_{1} \delta, \quad t \in \delta_{i} .
$$

Taking into account the inequality

$$
\left|K^{h}\left(\tau_{i}\right)-\xi_{3 i}^{h}\right| \leq h
$$

(see (5)) and the rule for choosing the control $I^{h}(\cdot)$ (36), we obtain for a.a. $t \in \delta_{i}$

$$
\nu_{4 i}(t) \leq C_{2} h, \quad C_{2}=2 \delta_{K} \max \left\{\left|I_{-}\right|,\left|I_{+}\right|\right\} .
$$

From (39)-41] it follows that for a.a. $t \in \delta_{i}$

$$
\nu_{i}(t) \leq 2 C_{1} \delta+C_{2} h+C_{3} \varepsilon_{1}\left(\tau_{i}\right) .
$$

Consequently, due to (38) and 42), we have for $i \in$ $\{0, \ldots, m-1\}$ the estimate

$$
\varepsilon_{1}\left(\tau_{i+1}\right) \leq\left(1+C_{3} \delta\right) \varepsilon_{1}\left(\tau_{i}\right)+C_{4} \delta(\delta+h) .
$$

From (43) and Lemma 3, we derive (for all $i \in$ $\{0, \ldots, m-1\})$ the inequalities

$$
\varepsilon_{1}\left(\tau_{i+1}\right) \leq \varepsilon_{1}(0)+C_{5}(\delta+h) .
$$

In this case,

$$
\varepsilon_{1}(t) \leq C_{*}(\delta+h) .
$$

Here $C_{*}>0$ is a constant that does not depend on $h, \delta$ and can be written explicitly. Now estimate the variation

$$
\varepsilon_{2}(t)=\left|M_{* 1}(t)-M_{1}^{h}(t)\right|^{2}, \quad t \in[0, \vartheta] .
$$

It is easily seen that for $t \in \delta_{i}=\left[\tau_{i}, \tau_{i+1}\right)$ the following inequality is true:

$$
\begin{aligned}
\varepsilon_{2}(t) \leq & \varepsilon_{2}\left(\tau_{i}\right)+\delta(h) \int_{\tau_{i}}^{t}\left|\dot{M}_{* 1}(\tau)-M_{1}^{h}(\tau)\right|^{2} \mathrm{~d} \tau \\
& +\int_{\tau_{i}}^{t} \mu_{i}^{(1)}(\tau) \mathrm{d} \tau \\
\mu_{i}^{(1)}(t)= & 2\left(M_{* 1}\left(\tau_{i}\right)-M_{1}^{h}\left(\tau_{i}\right)\right)\left(\dot{M}_{* 1}(t)-\dot{M}_{1}^{h}(t)\right)
\end{aligned}
$$

for $t \in \delta_{i}$. Note that

$$
\begin{gathered}
\dot{M}_{* 1}(t)=E_{1}\left(t, K_{*}\right)\left(1-\mu_{*}(t)\right)-\delta_{M} M_{* 1}(t), \\
\dot{M}_{1}^{h}(t)=E_{1}\left(t, K^{h}\right)\left(1-\mu^{h}(t)\right)-\delta_{M} M_{1}^{h}(t), \\
\left|\dot{K}^{h}(t)\right| \leq C_{0}
\end{gathered}
$$

for a.a. $t \in[0, \vartheta]$. Consider the value $\mu_{i}(t)$. By virtue of (47), we have for $t \in \delta_{i}$

$$
\mu_{i}(t)=\sum_{j=1}^{6} \lambda_{j i}(t), \quad t \in \delta_{i},
$$

where

$$
\begin{aligned}
\lambda_{1 i}(t) & =2 \beta_{i}^{(2)}\left(E_{1}\left(\tau_{i}, K_{*}\right)-E_{1}\left(t, K_{*}\right)\right) \mu_{*}(t), \\
\lambda_{2 i}(t) & =2 \beta_{i}^{(2)}\left(E_{1}\left(t, K_{*}\right)-E_{1}\left(t, K^{h}\right)\right), \\
\lambda_{3 i}(t) & =2 \beta_{i}^{(2)}\left(E_{1}\left(t, K^{h}\right)-E_{1}\left(\tau_{i}, K^{h}\right)\right) \mu^{h}(t), \\
\lambda_{4 i}(t) & =2 \delta_{M} \beta_{i}^{(2)}\left(M_{1}^{h}(t)-M_{* 1}(t)\right), \\
\lambda_{5 i}(t) & =2 \beta_{i}^{(2)} E_{1}\left(\tau_{i}, K_{*}\right)\left(\mu^{h}(t)-\mu_{*}(t)\right), \\
\lambda_{6 i}(t) & =2 \beta_{i}^{(2)}\left(E_{1}\left(\tau_{i}, K^{h}\right)-E_{1}\left(\tau_{i}, K_{*}\right)\right) \mu^{h}(t), \\
\beta_{i}^{(2)} & =M_{* 1}\left(\tau_{i}\right)-M_{1}^{h}\left(\tau_{i}\right) .
\end{aligned}
$$

Now, we estimate each term on the right-hand side of the equality (49). From (2), (3) and (48), it follows that

$$
\begin{aligned}
& \lambda_{1 i}(t) \leq C_{6}\left(\delta+\omega_{\varrho}(\delta)\right), \\
& \lambda_{3 i}(t) \leq C_{7}\left(\delta+\omega_{\varrho}(\delta)\right), \quad t \in \delta_{i},
\end{aligned}
$$




$$
\begin{aligned}
& \left|E_{1}\left(t, K_{*}\right)-E_{1}\left(t, K^{h}\right)\right| \\
& \quad \leq \varrho(t)\left|K_{*}^{\gamma}(t)-\left(K^{h}\right)^{\gamma}(t)\right|, \quad t \in[0, \vartheta],
\end{aligned}
$$

where

$$
\varrho(t)=\beta \sigma(t) A(t) L^{1-\gamma}(t),
$$

$\omega_{\varrho}(\delta)$ is the continuity modulus of $\varrho(t)$, i.e.,

$$
\omega_{\varrho}(\delta)=\sup \{|\varrho(t)-\varrho(t+\delta)|: t, t+\delta \in[0, \vartheta]\} .
$$

By virtue of Condition 3, we obtain

$$
\begin{aligned}
\left|K_{*}^{\gamma}(t)-\left(K^{h}\right)^{\gamma}(t)\right| & \leq K_{*}^{\gamma}(t)\left|1-\left(K^{h}(t) / K_{*}(t)\right)^{\gamma}\right| \\
& \leq\left(C^{(2)}\right)^{\gamma}\left|1-\left(1+\psi^{h}(t)\right)^{\gamma}\right|,
\end{aligned}
$$

where

$$
\psi^{h}(t)=\frac{K^{h}(t)-K_{*}(t)}{K_{*}(t)} .
$$

Taking into account (45), we conclude that the estimate

$$
\left|\psi^{h}(t)\right| \leq \frac{\varepsilon_{1}^{1 / 2}(t)}{C^{(1)}} \leq \frac{C_{*}^{1 / 2}(h+\delta)^{1 / 2}}{C^{(1)}}
$$

is valid. Let us choose $\delta_{*} \in(0,1)$ and $h_{*} \in(0,1)$ in such a way that the inequality

$$
\frac{C_{*}^{1 / 2}}{C^{(1)}}\left(h_{*}^{1 / 2}+\delta_{*}^{1 / 2}\right)<1
$$

is valid. Then, for $h \in\left(0, h_{*}\right)$ and $\delta \in\left(0, \delta_{*}\right)$ we have

$$
\sup _{t \in[0, \vartheta]}\left|K_{*}^{\gamma}(t)-\left(K^{h}\right)^{\gamma}(t)\right| \leq C_{8}\left(h^{1 / 2}+\delta^{1 / 2}\right),
$$

where the constant $C_{8}$ does not depend on $h$ and $\delta$. Using (51) and (52), we obtain for $\delta_{*} \in(0,1)$ and $h_{*} \in(0,1)$

$$
\begin{aligned}
& \lambda_{2 i}(t) \leq C_{9}\left(h^{1 / 2}+\delta^{1 / 2}\right), \\
& \lambda_{6 i}(t) \leq C_{10}\left(h^{1 / 2}+\delta^{1 / 2}\right), \quad t \in \delta_{i} .
\end{aligned}
$$

Furthermore, we have for $t \in \delta_{i}$

$$
\begin{aligned}
& \lambda_{4 i}(t) \leq C_{11} \varepsilon_{2}\left(\tau_{i}\right)+C_{12} \delta, \\
& \lambda_{5 i}(t) \leq \lambda_{i}^{(1)}(t)+\lambda_{i}^{(2)}(t),
\end{aligned}
$$

where

$$
\begin{aligned}
\lambda_{i}^{(1)}(t)= & 2 E_{1}\left(\tau_{i}, K_{*}\right)\left(M_{1 *}\left(\tau_{i}\right)-\tilde{u}_{*}^{h}\left(\tau_{i}\right)\right) \\
& \times\left(\mu^{h}(t)-\mu_{*}\left(\tau_{i}\right)\right) \\
\lambda_{i}^{(2)}(t)= & 4\left|M_{1}^{h}\left(\tau_{i}\right)-\tilde{u}_{*}^{h}\left(\tau_{i}\right)\right|\left|E_{1}\left(\tau_{i}, K_{*}\right)\right| f, \quad t \in \delta_{i}, \\
f= & \max \left\{\left|f_{-}\right|,\left|f_{+}\right|\right\} .
\end{aligned}
$$

Using the rule of forming the control $\mu^{h}(\cdot)$ (see (37)), we get

$$
\lambda_{i}^{(1)}(t) \leq 0 \quad \text { for a.a. } t \in \delta_{i} .
$$

In addition, from Theorem 1 we derive

$$
\lambda_{i}^{(2)}(t) \leq C_{13} \nu(h, \delta(h), \alpha(h)) \quad \text { for } t \in \delta_{i} .
$$

Taking into account (46), (49), (50), (53)-(57), we conclude for $t \in\left[\tau_{i}, \tau_{i+1}\right]$ that

$$
\begin{aligned}
\varepsilon_{2}(t) \leq & \varepsilon_{2}\left(\tau_{i}\right)\left(1+C_{14} \delta\right) \\
& +C_{15}\left(h^{1 / 2}+\delta^{1 / 2}\right) \delta \\
& +C_{16} \nu(h, \delta(h), \alpha(h)) \delta .
\end{aligned}
$$

Using Lemma 3 , we have for $i \in\{0, \ldots, m\}$

$$
\begin{aligned}
\varepsilon_{2}\left(\tau_{i}\right) \leq & \varepsilon_{2}(0)+C_{17}\left(h^{1 / 2}\right. \\
& \left.+\delta^{1 / 2}+\nu(h, \delta(h), \alpha(h))\right) .
\end{aligned}
$$

Then we get for $t \in[0, \vartheta]$

$$
\varepsilon_{2}(t) \leq C_{18}\left(h^{1 / 2}+\delta^{1 / 2}+\nu(h, \delta(h), \alpha(h))\right) .
$$

The validity of the theorem follows from (45) and (59).

Note that proving Theorem 2 we actually prove the convergence of the algorithm and not (time) convergence of the estimates.

Concluding, we describe the algorithm for the problem under consideration. Thus, we have the system (2) with the control $u=\{\mu, I\}$ and the system (3) with the unknown control $u_{*}=\left\{\mu_{*}, I_{*}\right\}$. We choose a family $\Delta_{h}=\left\{\tau_{i, h}\right\}_{i=0}^{m_{h}}$ of partitions of the interval $[0, \vartheta]$ with a step $\delta(h)=\tau_{i+1, h}-\tau_{i, h}$ and a function $\alpha(h)$ : $(0,1) \rightarrow(0,1)$ depending on the parameter $h$. The family $\Delta_{h}$ and the function $\alpha(h)$ satisfy Condition 2. Before the algorithm starts, we fix some value of measurement accuracy $h$, a partition $\Delta=\Delta_{h}$ and a number $\alpha=\alpha(h)$. The work of the algorithm is decomposed into $m-1$, $m=m_{h}$, identical steps. At the $i$-th step carried out during the time interval $\delta_{i}=\left[\tau_{i}, \tau_{i+1}\right), \tau_{i}=\tau_{i, h}$, the following actions are made. First, at the moment $\tau_{i}$, using the state $w^{h}\left(\tau_{i}\right)$ of the model (34), the result $\xi_{i}^{h}$ (satisfying the inequality (5)) of calculating the state of the system (2), we determine three numbers, namely, $v_{i}^{h}$ and $u_{i}^{h}=$ $\left\{\mu^{h}\left(\tau_{i}\right), I^{h}\left(\tau_{i}\right)\right\}$, by the formulas (35)-37). Then, during the time interval $\delta_{i}$, the constant control $v^{h}(t)=v_{i}^{h}$ is fed to the input of the model (34) and the constant control $u^{h}(t)=u_{i}^{h}$ is fed to the system (2), respectively. After these operations, at the moment $\tau_{i+1}$ the model state is recalculated (instead of the number $w^{h}\left(\tau_{i}\right)$, the number $w^{h}\left(\tau_{i+1}\right)=w^{h}\left(\tau_{i+1} ; w^{h}\left(\tau_{i}\right), v_{i}^{h}\right)$ is found; in addition, the vector $\xi_{i+1}^{h}$ is determined). Analogous actions are performed till the moment $\tau_{m_{h}-1, h}$.

As follows from Theorem 2, if the fixed measurement accuracy $h$ is sufficiently small, then the algorithm described above for forming the control $u(\cdot)$ in the system (2) provides the "tracking" (in uniform metric) of the solution $x_{*}(\cdot)$ of the system (3) by the 
solution $x^{h}(\cdot)$ of the system (2). Thus, the algorithm solves the problem of robust control.

Remark 1. Let us briefly dwell on the reasons that made us introduce Conditions 1-3. The appearance of Condition 1 is caused by the two factors: first, the "physical" (Nordhaus, 1994, Chapt. 2) sense of function $F(t)$ containing as a summand the value $4.1 \log _{2}(1+$ $\left.M_{1}(t) / 590\right)$ and, second, the necessity of boundedness in $L_{\infty}([0, \vartheta] ; \mathbb{R})$ of the function $\dot{\tilde{u}}(\cdot)$ (see the proof of Lemma 2). Condition 2 is necessary to prove Theorem 2 . Only under this condition does the right-hand side of the inequality (59) tend to zero as $h \rightarrow 0$. Condition 3 (namely, constants $C^{(1)}$ and $C^{(2)}$ from this condition) is used to obtain inequality (52).

\section{Results of computer modeling}

The algorithm described above was tested on a computer. In Figs. 2-5, the results of computer modeling are presented for the following case:

$$
\begin{aligned}
c_{1} & =c_{2}=c_{3}=1, & \sigma & =1+0.5 t, \\
c_{4} & =0.5, & O(t) & =5 \sin t, \\
\delta_{K} & =0.65, & L(t) & =1, \\
a_{1} & =30, & a_{2} & =60, \\
\delta_{M} & =0.0833, & \mu_{*}(t) & =1+0.5 t, \\
\beta & =0.1, & I_{*}(t) & =1+0.15 t^{2}, \\
\gamma & =0.1, & A(t) & =2 t^{1 / 2} .
\end{aligned}
$$

The parameters are as follows:

$$
f_{-}=1, \quad f_{+}=10, \quad I_{-}=1, \quad I_{+}=2 .
$$

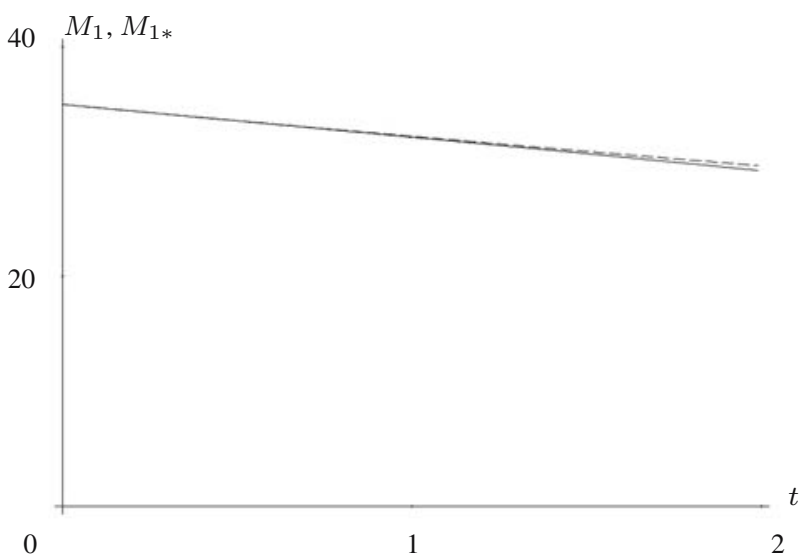

Fig. 2. Trajectories $M_{1}(t)$ (dashed line) and $M_{1 *}(t)$ (solid line) in the case of $\delta=0.0001, h=0.001$.

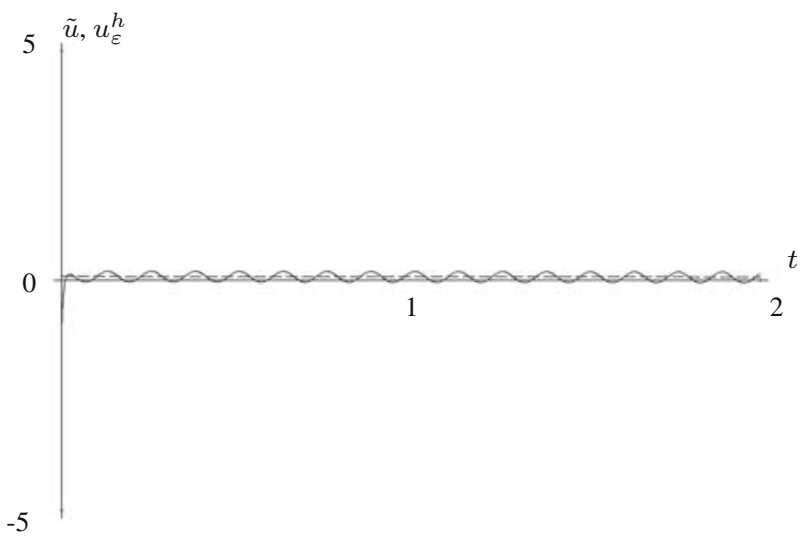

Fig. 3. Function $\tilde{u}(t)$ (solid line) and control $u_{\varepsilon}^{h}(t)$ (dashed line) in the case of $\delta=0.0001, h=0.001$.

The initial conditions for the system are the following:

$$
\begin{aligned}
T_{0}(0) & =1, & & T_{1}(0)=0.5, \\
M_{1}(0) & =35, & & K(0)=5 .
\end{aligned}
$$

Equations (2) and (3) were solved by the Euler method with step $\delta$. During the experiment, we assume

$$
\begin{aligned}
\xi_{1 i}^{h} & =T_{0}\left(\tau_{i}\right)+h \sin (50 t), \\
\xi_{2 i}^{h} & =T_{1}\left(\tau_{i}\right)+h \sin (50 t), \\
\xi_{3 i}^{h} & =K\left(\tau_{i}\right)+h \sin (50 t), \quad \alpha=0.1 .
\end{aligned}
$$

In Figs. 2 and 4, trajectories $M_{1}(t)$ (dashed line) and $M_{1 *}(t)$ (solid line) are presented. In Figs. 3 and 5, control $u_{\varepsilon}^{h}(t)$ (dashed line) and function

$$
\tilde{u}(t)=\log _{2}\left(1+\frac{M_{1}(t)}{590}\right)
$$

(solid line) are presented. Figures 2 and 3 correspond to the case of $\delta=0.0001, h=0.001$; Figs. 4 and 5 correspond to the case of $\delta=0.01, h=0.5$.

\section{Conclusions}

A control problem for a fourth order system was considered. The problem consists in constructing an algorithm of stable formation of control characteristics providing a given quality of the process under conditions of measuring a part of the phase coordinates and the action of uncontrolled disturbances. On the basis of the feedback control method, a solving algorithm (the reconstruction-control controller) is designed. The algorithm consists of two blocks: the block of online reconstruction and the block of positional control.

\section{Acknowledgment}

The work of V. Maksimov was supported in part by the RFBR (projects 13-01-00110a, 13-01-12446-ofi-m2), 


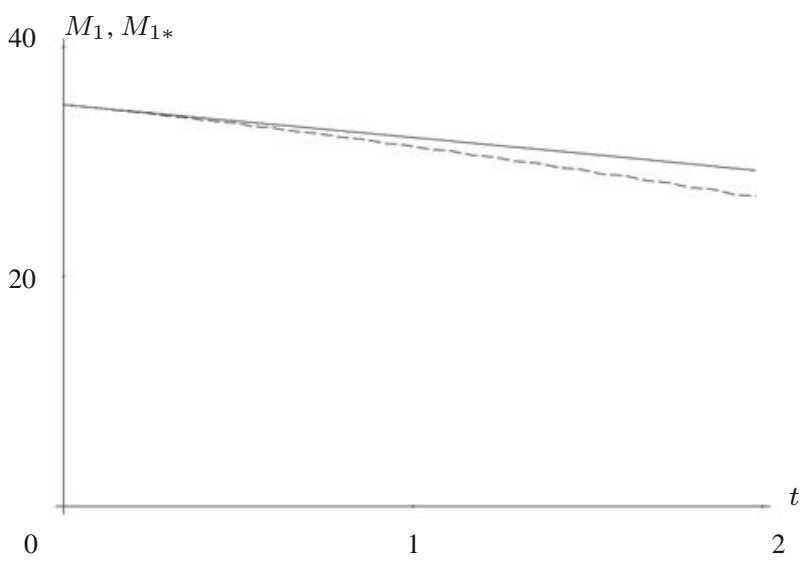

Fig. 4. Trajectories $M_{1}(t)$ (dashed line) and $M_{1 *}(t)$ (solid line) in the case of $\delta=0.01, h=0.5$.

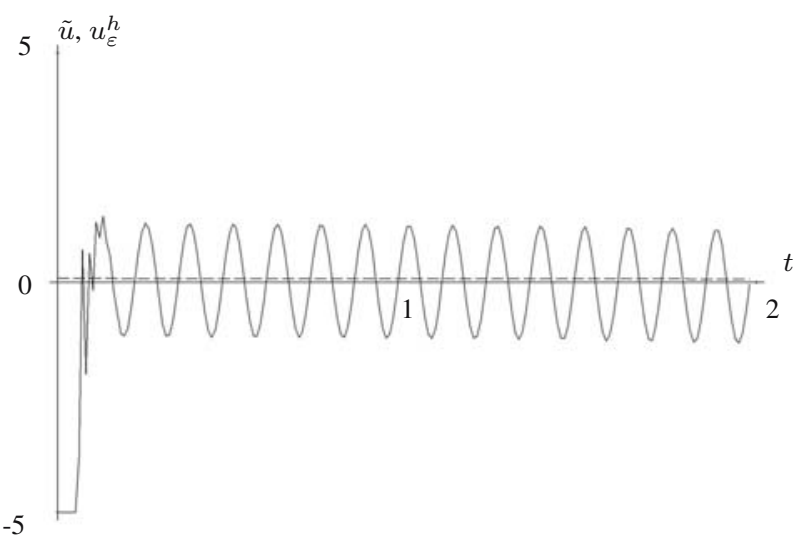

Fig. 5. Function $\tilde{u}(t)$ (solid line) and control $u_{\varepsilon}^{h}(t)$ (dashed line) in the case of $\delta=0.01, h=0.5$.

by the Russian Fund for Humanities (14-02-00331), by the Programs of Basic Research of the Presidium of the Russian Academy of Sciences (project 12-P-6-1038), and by the Program for Support of Leading Scientific Schools of Russia (6512.2012.1).

\section{References}

Blizorukova, M.S.and Maksimov, V.I. (2006). A control problem under incomplete information, Automation and Remote Control 67(3): 131-142.

Blizorukova, M.S. and Maksimov, V.I. (2010). On some dynamical reconstruction problems for a nonlinear system of the second-order, Opuscula Mathematica 30(4): pp. 399-410.

Keesman, K.J. and Maksimov, V.I. (2008). On feedback identification of unknown characteristics: A bioreactor case study, International Journal of Control 81(1): 134-145.

Krasovskii, N.N. and Subbotin, A.I. (1988). Game-Theoretical Control Problems, Springer Verlag, New York, NY/Berlin.
Maksimov, V. (2010). On one algorithm for solving the problem of source function reconstruction, International Journal of Applied Mathematics and Computer Science 20(2): 239-247, DOI: 10.2478/v10006-010-0017-3.

Maksimov, V.I. (2011). The tracking of the trajectory of a dynamical system Journal of Applied Mathematics and Mechanics 75(6): 667-674.

Maksimov, V.I. (2013). On reconstruction of controls in uniform metric Journal of Applied Mathematics and Mechanics 77(2): 292-301.

Nordhaus, W.D. (1994). Managing the Global Commons. The Economics of Climate Change, MIT Press, Cambridge, MA/London.

Osipov, Yu.S. and Kryazhimskii, A.V. (1995). Inverse Problems of Ordinary Differential Equations: Dynamical Solutions, Gordon and Breach, London.

Osipov, Yu.S., Kryazhimskii, A.V. and Maksimov, V.I. (2011). Methods of Dynamical Reconstruction of Inputs of Control Systems, UB RAS, Ekaterinburg, (in Russian).

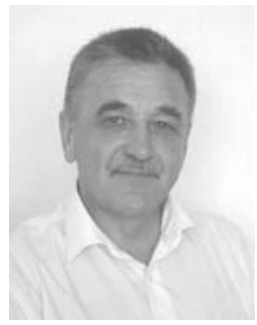

Vladimir Kapustyan graduated in applied mathematics from the Dnipropetrovsk National University of Railway Transport, Dnipropetrovsk, Ukraine, in 1975. He received the candidate as well as doctor of physics and mathematics degrees from the Taras Shevchenko National University of Kyiv, in 1982 and 1994, respectively. From 1996 to 2003 he was the head of the Department of Computer and Information Technologies at the Dnipropetrovsk National University of Railway Transport. Since 2003, he has been the head of the Department of Mathematical Modeling for Economic Systems at the National Technical University of Ukraine, Kyiv. He is the author of more than 100 technical publications, including two monographs. His research interests include control theory, distributed parameter systems and mathematical modeling. Doctor Kapustyan has been on editorial boards of a number of journals.

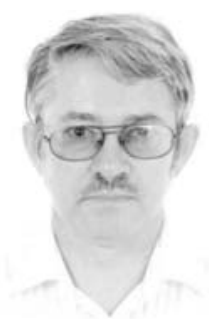

Vyacheslav Maksimov graduated in mathematics and mechanics from Ural State University, Ekaterinburg, Russia, in 1972. He received the candidate as well as doctor of physics and mathematics degrees from the Institute of Mathematics and Mechanics, Ural Branch of the Russian Academy of Sciences, in 1978 and 1992, respectively. Since 1972, he has been with the Institute of Mathematics and Mechanics, Ekaterinburg. Since 1994, he has been the head of a department at the same institute and a professor at the Chair of Control Systems Modeling of Ural Federal University, Ekaterinburg. He is the author of more than 100 technical publications, including three monographs. His research interests are primarily focused on control theory, distributed parameter systems and mathematical modeling. Doctor Maksimov is a member of the American Mathematical Society and of the IFIP WG7.2. He has also been a member of editorial boards of various journals.

Received: 8 April 2013

Revised: 20 June 2013 